\title{
Les stations d'une passion
}

\section{Sandra Ziegler}

PhD, MS, directrice des EMH depuis juin 2018

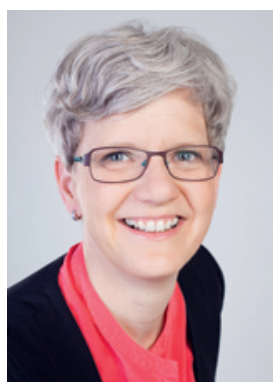

Au début de l'été 1998, alors que je me trouvais au centre-ville de Saint-Gall, j'ai eu la certitude de m'être trouvé une passion. C'était pendant la campagne de votation de l'initiative "pour la protection génétique» et en tant que doctorante en biologie cellulaire, je m'investissais aux stands pour rendre les concepts, outils et chances de la technique génétique compréhensibles pour les passants. L'initiative a été rejetée, nous faisions partie des gagnants. Mais en ce qui me concernait, une découverte sur le plan personnel pesait presque plus lourd dans la balance. J'avais adoré décomposer ces contenus scientifiques complexes, les transposer dans un nouveau contexte quotidien, les rendre accessibles autrement. Je voulais poursuivre sur cette voie.

J'avais adoré décomposer ces contenus scientifiques complexes, les transposer dans un nouveau contexte quotidien, les rendre accessibles autrement.

J'ai ensuite dirigé le petit musée scientifique du $p s i$ forum de l'Institut Paul Scherrer (PSI) et transmis sur la base d'expositions et de visites guidées les sujets PSI, de la recherche énergétique aux piles à combustible, de l'accélérateur de particules à la protonthérapie contre les tumeurs oculaires. Je pense que nous avons pu toucher et passionner énormément de gens au PSI avec ces activités, mais que nous faisions partie des pionniers en Suisse. Hors de Suisse, et plus particulièrement dans les pays anglo-saxons, les activités de communication scientifique étaient déjà beaucoup plus établies, l'expérience acquise beaucoup plus grande. Aidée d'une bourse du Fonds national suisse, j'ai donc décidé de suivre des études de journalisme scientifique et de relations publiques à l'Université de Boston. Après ce cursus, forte d'idées précieuses et de nombreuses impulsions nouvelles, j'ai dirigé depuis Boston la communication interne mondiale de l'unité de recherche NIBR de Novartis.
De retour en Suisse, j’ai retrouvé un environnement de recherche universitaire. J'ai communiqué les dernières découvertes en termes de protection des eaux ou des solutions à des problèmes sanitaires dans des pays en voie de développement pour l'institut de recherche sur l'eau EAWAG, et créé et développé ces dernières années les activités de communication du Friedrich Miescher Institut de Bâle sur le Web et les réseaux sociaux, mais également dans les médias classiques.

Toutes ces tâches mettaient l'accent sur la manière de rendre des thèmes parfois très complexes attrayants pour les différents publics cibles: chercheurs et profanes, personnel dans divers pays, hommes et femmes politiques, journalistes. Quels sont les besoins des différentes personnes, quel est leur bagage, qu'est-ce qui est pertinent pour elles? Je continue d'y réfléchir de mettre au point des stratégies et de les appliquer de diverses manières avec enthousiasme.

En tant que nouvelle directrice des EMH, j'apporte à l'équipe ma passion et mon expérience dans ce domaine. Je considère aussi notre mission de ce point de vue: en tant que maison d'édition indépendante sur le plan rédactionnel, nous présentons des contenus attrayants et pertinents sous différents formats aux médecins suisses. Nous veillons, avec le personnel médical des rédactions, à proposer une formation continue

Je me réjouis à la perspective de la collaboration au sein des EMH et avec les différents représentants du secteur de la santé.

de premier ordre et renforçons le dialogue entre les différents acteurs et entre pairs au travers d'articles de haut niveau. Je me réjouis à la perspective de la collaboration au sein des EMH et avec les différents représentants du secteur de la santé et j'ai hâte de relever les défis liés à l'environnement concurrentiel dans lequel les EMH évoluent. 